\title{
RECONFIGURABLE CONTROL DESIGN WITH INTEGRATION OF A REFERENCE GOVERNOR AND RELIABILITY INDICATORS
}

\author{
Philippe WEBER, Boumedyen BOUSSAID, Ahmed KHELASSI, Didier THEILliOL, \\ CHRISTOPHE AUBRUN
}

\author{
Research Centre for Automatic Control of Nancy, CRAN CNRS UMR 7039 \\ University of Lorraine, BP 70239, 54506 Vandœuvre Cedex, France \\ e-mail: \{philippe.weber, ahmed.khelassi\}@eran.uhp-nancy.fr \\ \{didier.theilliol, christophe.aubrun\} @cran.uhp-nancy.fr
}

\begin{abstract}
A new approach to manage actuator redundancy in the presence of faults is proposed based on reliability indicators and a reference governor. The aim is to preserve the health of the actuators and the availability of the system both in the nominal behavior and in the presence of actuator faults. The use of reference governor control allocation is a solution to distribute the control efforts among a redundant set of actuators. In a degraded situation, a reconfigured control allocation strategy is proposed based on on-line re-estimation of the actuator reliability. A benefit of incorporating reliability indicators into over-actuated control system design is the smart management of the redundant actuators and improvement of the system safety. Moreover, when the fault is severe, an adaptation approach using the reference governor is proposed. The reference governor unit is a reference-offset governor based on a discrete-time predictive control strategy. The idea is to modify the reference according to the system constraints, which become stricter after the occurrence of an actuator fault. The proposed approach is illustrated with a flight control application.
\end{abstract}

Keywords: fault tolerant control systems, control allocation, reliability estimation, performance degradation, reference governor.

\section{Introduction}

In order to satisfy the increased economic demand for high plant availability and system safety, dependability is becoming an essential need in industrial automation. In this context and to satisfy these requirements, Fault-Tolerant Control (FTC) is introduced. The aim of FTC is to keep a plant available by the ability to achieve the objectives that have been assigned in the faulty behavior and accept reduced performance when critical faults occur (Blanke et al., 2006; Noura et al., 2009). In most safety critical systems, actuator redundancy is often used, e.g., in the three major control effectors in aircraft flight control (aileron, elevator and rudder). They are usually designed utilizing one control effector or actuator for each rational degree of freedom. However, due to the increased requirements on the reliability, maneuverability and survivability of modern and future aircraft, control effectors are no longer limited to these three conventional control effectors and many more control actuators have been introduced.

Moreover, several tools and approaches have been proposed to manage redundancy and to distribute the de- sired control efforts among a set of actuators. A common approach is to use optimal control theory to shape the closed-loop dynamics and to distribute the desired control efforts in one step. Optimized methods like linear quadratic control (Kawakernaak and Sivan, 1972) and robust control (Zhou et al., 1996) are readily available. An alternative strategy is to separate the regulation task from control distribution. Indeed, the control law specifies only the desired control efforts to be produced, and a separate control allocation module is introduced in the control loop to distribute the control among the actuators (Harkegard, 2003). This strategy is used in practical applications in aerospace control.

In the work of Durham (1993), control allocation is applied to several airplane flights. Bordignon (1996) illustrates this technique for an F-18 fighter with seven independent moments. A review of existing methods can be found in the work of Enns (1998). In the degraded functional, reconfigurable control allocation is employed. The advantage of this strategy is the ability to accommodate the control surface damages without modifying the 
controller parameters. Various approaches to control reallocation have been proposed for flight control systems. Alwi and Edwards (2008) propose an on-line control allocation with a sliding modes controller. Burken et al. (2001) reformulate the control allocation problem based on a quadratic programming problem. Reconfigurable control allocation based on pseudo-inverse methods is illustrated by Zhang et al. (2007). Recently, Johansen and Johansen (2008) as well as Casavola and Garone (2010) have proposed an adaptive control allocation approaches.

The main goal of these methods is to improve the safety and reliability of the system, which is rarely associated with an objective criterion that guides a design (Theilliol et al., 2009). Some works have introduced reliability analysis for fault-tolerant control systems in order to take into account the health of the actuators in the reconfiguration strategy (Guenab et al., 2006). Reconfigurability analysis was investigated for a reliable faulttolerant control design by Khelassi et al. (2009). In this context, a reconfigurable control allocation design with integration of the reference governor is proposed in this paper based on actuator reliability. The aim is to smartly manage the redundant actuators in order to satisfy the performance requirements and improve the probability of the success of the mission.

Modification of the control law following the reallocation step moves the faulty operating point to a degraded one. Under this operating point, some problems may occur due to physical limitations inherent to the system. In particular, FTC must take into consideration the input/state constraints which are dictated by actuator saturations and keep plant variables within safe limits. In recent years, several feedback control techniques of dynamic systems have been developed which are able to handle input and/or state-related constraints (Gilbert and Tan, 1991; Gilbert et al., 1995; Bemporad et al., 1997; Angeli et al., 2001; Theilliol et al., 2008a; Theilliol et al., 2008b), using methods based on predictive control ideas, to synthesize the command or the Reference Governor (RG).

Kolmanovsky and Sun (2006) propose a Parameter Governor (PG) unit which enforces pointwise-in-time constraints on the evolutions of relevant system variables. Later, both RG and PG actions are integrated in a single unit as the Reference-Offset Governor (ROG) (Casavola et al., 2007), which adds many advantages especially in enlarging the set of feasible evolutions of the system. The function of the ROG device is to modify, whenever necessary, the reference and add an offset to the nominal control action in order to enforce pointwise-in-time constraints and to improve the overall system transient performance (cf. Casavola et al., 2006; 2007). The adaptation of the ROG with fault tolerant control systems is presented by Boussaid et al. (2010).

The paper is organized as follows. The reconfigurable control allocation and the reference governor issue for actuator faults are presented in Section 2. The reformulation of the reconfigurable control allocation problem integrating the reliability requirements and the reference adaptation is proposed in Section 3. In Section 4, the proposed approach is illustrated and applied to a linearized aircraft model from the ADMIR simulator. Finally, concluding remarks are given in the last part of this work.

\section{Reconfigurable control allocation and reference governor design}

The proposed reconfigurable control system is composed of three modules: the reference governor module, the nominal controller and the allocation module. The benefit of such a structure (e.g., Fig. 1) is the possibility to deal with the largest range of faults that may occur in the system. For minor faults, the reconfiguration of the allocation module can compensate the effects of faults. Nevertheless, major faults should be treated carefully such as the saturation of the actuators. Thus, the reference governor is one solution to deal with actuator saturation by modifying the references to acceptable performances.

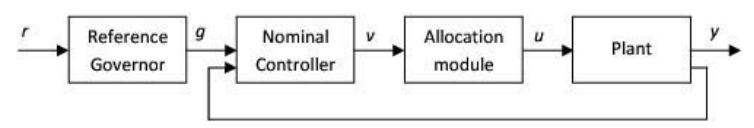

Fig. 1. Simplified structure of the proposed nominal system.

2.1. LTI formulation of control allocation. Let us consider the LTI system given by

$$
\left\{\begin{aligned}
x(t+1) & =A x(t)+B u(t), \\
y(t) & =C x(t),
\end{aligned}\right.
$$

where $A \in \mathbb{R}^{n \times n}, B \in \mathbb{R}^{n \times m}$ and $C \in \mathbb{R}^{p \times n}$ are respectively the state, the control and the output matrices. $x \in \mathbb{R}^{n}$ is the system state, $u \in \mathbb{R}^{m}$ is the control input, $y \in \mathbb{R}^{p}$ is the system output, and $(A, B)$ is stabilizable. Control allocation is generally used for over-actuated systems, where the number of operable controls is greater than the controlled variables.

As defined by Harkegard and Glad (2005), while $\operatorname{rank}(B)=q<m$ is satisfied, a virtual control input $v \in \mathbb{R}^{q}$ can be introduced and an equivalent description of (1) can be obtained as follows:

$$
\left\{\begin{aligned}
x(t+1) & =A x(t)+B_{v} v(t), \\
v(t) & =B^{*} u(t), \\
y(t) & =C x(t)
\end{aligned}\right.
$$

by considering the factorization

$$
B=B_{v} B^{*},
$$


where $B_{v} \in \mathbb{R}^{n \times q}$ and $B^{*} \in \mathbb{R}^{q \times m}$.

In fact, $v(t)$ represents the set of the desired control efforts to be applied to the system in order to satisfy the performance requirements. It can be defined by solving based on the following linear quadratic optimization problem:

$$
v(t) \rightarrow \min _{u} \int_{0}^{\infty}\left(x(t)^{T} Q_{2} x(t)+v(t)^{T} R_{2} v(t)\right) \mathrm{d} t,
$$

where $Q_{2} \in \mathbb{R}^{n \times n}$ and $R_{2} \in \mathbb{R}^{q \times q}$.

For simplicity and for this study, the case $q=p$, i.e., when the number of virtual controls equals the number of variables to be controlled, is considered.

Property 1. If the matrix $B$ is not full rank, $\operatorname{rank}(B)=$ $q<n$, it can be factorized as $B=B_{v} B^{*}$, where $B_{v} \in \mathbb{R}^{n \times q}, B^{*} \in \mathbb{R}^{q \times m}$ and $\operatorname{rank}\left(B^{*}\right)=q$. The virtual control input $v(t)$ can be determined in this case as $v(t)=B^{*} u(t)$, where $B^{*}=\left(B_{v}^{T} B_{v}\right)^{-1} B_{v}^{T} B$

The control allocation problem can be expressed as a constrained linear mapping one based on the relationship,

$$
\begin{gathered}
v(t)=B^{*} u(t), \\
u_{\min } \leq u \leq u_{\max },
\end{gathered}
$$

where (6) is physical actuator saturation.

Optimization based control allocation methods aim at finding an optimal solution. If there is no exact solution, the optimal control is the feasible one such that $B^{*} u(t)$ approximates $v(t)$ as well as possible. The optimal control input can be obtained by a two-step optimization, namely, sequential quadratic programming:

$$
\begin{gathered}
\mathcal{U}=\underset{u_{\min } \leq u \leq u_{\max }}{\arg \min }\left\|B^{*} u-v\right\|_{2}, \\
u=\arg \min _{u \in \mathcal{U}}\left\|W_{u} u\right\|_{2},
\end{gathered}
$$

where $\mathcal{U}$ is the set of feasible solutions subject to the cost criterion (8). The weighting matrix $W_{u} \in \mathbb{R}^{m \times m} \succ 0$ is used to give a specific priority level to the actuators.

In order to improve the safety of the system and preserve the actuators, a specific choice of the weighting matrix $W_{u}$ is proposed based on actuator reliability indicators. The weighing matrix $W_{u}$ is considered a key to manage the redundant actuators and contribute to a reliable controller improving system reliability. This technique can increase the life time of the system and prevent additional faults from occurring.

2.2. Reliability integration in control allocation design. Reliability $R(t)$ is defined as the probability that units, components, equipments and systems will accomplish the intended function for a specified period of time under some stated conditions and specific environments (Gertsbakh, 2000).

In many situations, and especially in the present study, failure rates are obtained from components under different load levels. Several mathematical models have been developed to define the failure level in order to estimate the failure rate $\lambda$ (Martorell et al., 2009). The proportional hazard model introduced by Cox (1972) is used in this paper.

Definition 1. The failure rate is modeled as follows:

$$
\lambda_{i}=\lambda_{i}^{b l} \mu_{i}(\ell, \vartheta)
$$

where $\lambda_{i}^{b l}$ represents the baseline failure rate (nominal failure rate) for the $i$-th subsystem or component and $\mu_{i}(\ell, \vartheta)$ is a function (independent of time) taking into account the effects of loads applied with $\ell$ presenting an image of the load and $\vartheta$ defining some parameters of the subsystem or component.

Definition 2. Different definitions of the load function $\mu_{i}(\ell, \vartheta)$ exist in the literature (Martorell et al., 2009). However, the exponential form is commonly used (Guenab et al., 2006). Moreover, the failure rate functions for the exponential distribution change according to the load level, assumed to be directly associated with the control input,

$$
\mu_{i}(\ell, \vartheta)=\mu\left(\left\|u_{i}\right\|\right)=\exp \left(\left\|u_{i}\right\|_{2}^{2}\right) .
$$

As given in (9), the failure rate of the actuator can be defined according to the load level, which is proportional to the control input applied,

$$
\lambda_{i} \geq \lambda_{i}^{b l}, \quad i=1, \ldots, m .
$$

Definition 3. For the exponential distribution of reliability, the mean time before the first failure (MTTF) can be adopted as a reliability indicator defined

$$
\mathrm{MTTF}=\int_{0}^{\infty} R(t) \mathrm{d} t=\frac{1}{\lambda}
$$

Moreover, for $m$ redundant components, the overall system reliability can be computed at the end of the mission defined by $t=t_{M}$ as follows:

$$
R_{g}\left(t_{M}\right)=1-\prod_{i=1}^{m}\left(1-R_{i}\left(t_{M}\right)\right) .
$$

To implement the solution of the control allocation problem, and keep the set of the actuators available as long as possible, the desired efforts $v(t)$ defined by the controller can be distributed proportionally to the actuator reliability indicator. In order to consider such an approach, the values of the weighing matrix $W_{u}$ are defined 
as

$$
W_{u}=\left(\begin{array}{cccc}
\frac{\lambda_{1}^{b l}}{\lambda_{\max }^{b l}} & & & 0 \\
& \frac{\lambda_{2}^{b l}}{\lambda_{\max }^{b l}} & & \\
& & \ddots & \\
0 & & & \frac{\lambda_{m}^{b l}}{\lambda_{\max }^{b l}}
\end{array}\right) \succ 0,
$$

where $\lambda_{\max }^{b l}=\max \left(\lambda_{i}^{b l}\right)$ is the upper failure rate corresponding to the least reliable actuator.

Consequently, for $\lambda_{i}^{b l} \ll \lambda_{\max }^{b l}, w_{i} \rightarrow 0$ and so the associated control component $u_{i}^{*}$ (a solution of the optimization problem (8) becomes very large. In addition, as $\lambda_{i}^{b l} \rightarrow \lambda_{\max }^{b l}, w_{i} \rightarrow 1$ and the associated control input is heavily weighted. The actuators are utilized in the control allocation proportionally to their health. This off-line synthesis of the control allocation strategy reduces the solicitation of the sensible actuators, which improves system safety and minimizes actuator aging. Indeed, the following relation can be achieved:

$$
\left\{\lambda_{i}^{b l} \rightarrow \lambda_{\max }^{b l}: u_{i}^{*} \rightarrow 0\right\},
$$

where $\lambda_{\max }^{b l}$ is the failure rate of the less reliable actuator.

2.3. LQ controller design. Let us consider the global system including the RG unit and the feedback controller, as depicted in Fig. 2 According to (2), and by

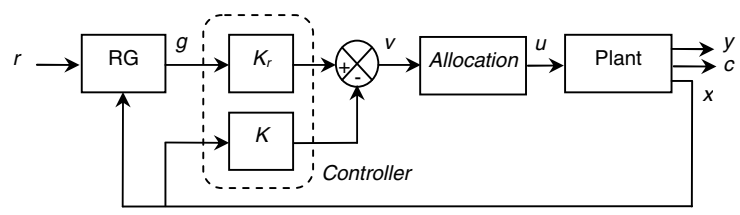

Fig. 2. Global system diagram including controller and reference governor blocks.

solving the Linear Quadratic Regulation (LQR) problem (Staroswiecki, 2003; Harkegard and Glad, 2005), the optimal control law is given by

$$
v(t)=-K^{*} x(t)+K_{r}^{*} g(t),
$$

where $g(t) \in \mathbb{R}^{p}$ is the manipulable reference, $K^{*}$ and $K_{r}^{*}$ the controller feedback and feed-forward gains, respectively, with

$$
\begin{aligned}
& K^{*}=R^{-1} B_{v}^{T} S, \\
& K_{r}^{*}=R^{-\frac{1}{2}}\left(C\left(B_{v} K^{*}-A\right)^{-1} B_{v} R^{-\frac{1}{2}}\right)^{+},
\end{aligned}
$$

where $Q \in \mathbb{R}^{n \times n}$ is a positive semi-definite matrix and $R \in \mathbb{R}^{q \times q}$ is a positive definite matrix. $Q$ and $R$ are preselected by the designer to achieve the nominal performance. $S$ is a unique positive semi-definite and symmetric solution of the Algebraic Riccati Equation (ARE)

$$
A^{T} S+S A+Q-S B_{v} R^{-1} B_{v}^{T} S=0 .
$$

2.4. LTI formulation of the reference governor. As mentioned in Section 2.1 and according to (5), the control input $u(t)$ can be written as

$$
u(t)=P v(t)
$$

with

$$
P=W_{u}^{-1}\left(B^{*} W_{u}^{-1}\right)^{+} .
$$

Replacing (16) in (20), we can write

$$
u(t)=-K x(t)+K_{g} g(t),
$$

where $K=P K^{*}, K_{g}=P K_{r}^{*}$, and $g(t) \in \mathbb{R}^{p}$ is the RG output, with $r(t) \in \mathbb{R}^{p}$ being the nominal reference.

Substituting (21) in (1), we obtain

$$
x(t+1)=\Phi x(t)+G g(t),
$$

where $\Phi=(A-B K)$ and $G=B K_{g}$. We deal only with actuator saturations in this work, which means that we consider only constraints on the control inputs. Set $H_{c}=-K$ and $L=K_{g}$, so that the constraint vector $c(t)$ can be written as:

$$
c(t)=H_{c} x(t)+L g(t) .
$$

Thus, the LTI system in (1) becomes

$$
\left\{\begin{aligned}
x(t+1) & =\Phi x(t)+G g(t), \\
y(t) & =H_{y} x(t), \\
c(t) & =H_{c} x(t)+L g(t),
\end{aligned}\right.
$$

$c(t) \in \mathbb{R}^{n_{c}}$ being the constraint vector, $c(t) \in \mathcal{C}$; for any $t \in \mathbb{Z}_{+}$, and $\mathcal{C} \subset \mathbb{R}^{n_{c}}$ is a prescribed constraint set.

We make the following assumptions:

A.1. $\Phi$ is a Stable matrix.

A.2. The system (24) is offset-free with respect to $g(t)$, i.e., $H_{y}\left(I_{n}-\Phi\right)^{-1} G_{g}=I_{p}$.

The RG design problem consists in generating, at each time $t$, the command input $g(t)$ as an algebraic function of the current state $x(t)$ and reference $r(t)$,

$$
g(t):=\bar{g}(x(t), r(t)) .
$$

The RG output is based on the minimization of a cost function subject to prescribed constraints. The cost func- 
tion has the following form:

$$
J(x(t), g(t), r)=\|g(t)-r\|_{\Psi_{g}}^{2},
$$

where $\Psi_{g}=\Psi_{g}^{T}>0_{m}$ and $\|v\|_{\Psi}^{2}:=v^{T} \Psi v$. Thus, at each time $t \in \mathbb{Z}_{+}$, the RG output is chosen according to the solution of the following constrained optimization problem (cf. Casavola et al., 2007):

$$
g(t):=\arg \min _{g \in \mathcal{V}(x(t))} J(x(t), g(t), r),
$$

where $\mathcal{V}(x(t))$ represents the set of all virtual sequences whose evolutions starting from $x(t)$ satisfy the constraints during transients and which is constructed from the recursive set developed by Casavola et al. (2007).

\section{On-line control re-allocation and reference governor synthesis}

In a degraded behavior and after a fault occurrence, the desired efforts are distributed among the actuators based on the re-estimation of their reliability indicators. The control inputs $u_{i}, i=1, \ldots, m$, are re-allocated taken into account actuator aging (see Fig. 3).

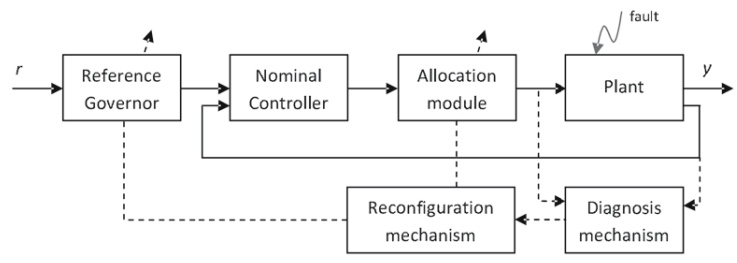

Fig. 3. Proposed structure of the reconfigurable control system.

Due to abnormal operation or material aging, actuator faults may occur and increase the complexity of the on-line control allocation problem. In this work, a loss of effectiveness control is considered where the system (2) can be written as a degraded functional as follows:

$$
\left\{\begin{aligned}
x(t+1) & =A x(t)+B_{v} v(t), \\
v(t) & =B_{f}^{*} u(t), \\
y(t) & =C x(t) .
\end{aligned}\right.
$$

The matrix $B_{f}^{*}$ can be written according to the nominal control input matrix $B^{*}$ and the control effectiveness factors $\gamma_{i} \in\left[\begin{array}{ll}0 & 1\end{array}\right], i=1, \ldots, m$, as follows:

$$
B_{f}^{*}=B^{*}\left(I_{m}-\Gamma\right), \quad \Gamma=\left(\begin{array}{cccc}
\gamma_{1} & & & 0 \\
& \gamma_{2} & & \\
& & \ddots & \\
0 & & & \gamma_{m}
\end{array}\right) \text {. }
$$

Indeed, if $\gamma_{i}=0$, then the $i$-th actuator is considered in the fault-free case. Nevertheless, when $0<\gamma_{i}<1$, a fault which presents a partial loss of effectiveness control is considered. Moreover, when $\gamma_{i}=1$, a failure is considered and the $i$-th actuator is out of order.

3.1. On-line reliability indicator estimation. The control redistribution in the faulty case requires the update of the weighting matrix and the estimation of the actuator reliability indicators according to the time of fault occurrence. In the following, a fault is assumed to be detected and isolated at time $t=t_{f}$. As presented previously, the MTTF is defined as the expected value of the failure distribution $V(t)$ :

$$
\mathrm{MTTF}=1+\sum_{t=0}^{\infty} \prod_{j=1}^{t}(1-\lambda(j)) .
$$

Indeed, for a reconfigurable system at $\tau=t_{f}+\Delta t$, the mean time before the first failure can be written according to $t=\tau$ as follows:

$$
\operatorname{MTTF}_{\tau}=1+\sum_{t=\tau}^{\infty} \prod_{j=1}^{t}(1-\lambda(j)),
$$

where $\mathrm{MTTF}_{\tau}$ can be seen as an estimate of the mean time before a failure for a new reconfiguration. The failure rate corresponding to the rest of the component life time $\lambda_{i}^{f}$ can be written as

$$
\lambda_{i}^{f}=\frac{\lambda_{i}^{0}}{\lambda_{i}^{0}(\tau-1) T e+1} \exp \left(\lambda^{0}(\tau-1) T_{e}\right),
$$

where $\lambda^{f}$ is calculated according to the load level defined for $t \in\left[0, t_{f}\right]$ as in (9) and $T_{e}$ is the sampling time.

In order to integrate actuator degradation in the reconfigured control allocation strategy, the control input can be obtained by solving the optimization problem (7) and (8) where, for $t \in\left[\tau, t_{M}\right], B^{*}$ is replaced by $B_{f}^{*}$ and the weighting matrix $W_{u}$ is re-estimated and changed online according to the new failure rates values $\lambda_{i}^{f}$,

$$
W_{u}=\left(\begin{array}{cccc}
\frac{\lambda_{1}^{f}}{\lambda_{\max }^{f}} & & & 0 \\
& \frac{\lambda_{2}^{f}}{\lambda_{\max }^{f}} & & \\
& & \ddots & \\
0 & & & \frac{\lambda_{m}^{f}}{\lambda_{\max }^{f}}
\end{array}\right) \succ 0 .
$$

In fact, $\lambda_{\max }^{f}=\max \left(\lambda_{i}^{f}\right), i=1, \ldots, m$, is the upper value of $\lambda_{i}^{f}$ corresponding to the most degraded actuator.

Indeed, if an actuator fault occurs, the weighing matrix will be changed on-line $W_{u}$ and a control input $u(t)$ 
is re-allocated smartly in order to minimize the use of the sensible actuators.

3.2. Pseudo-inverse method. In the faulty case, the control re-allocation problem consist in finding the control input $u(t)$ minimizing (8) and satisfying $B_{f}^{*} u(t)=v(t)$. If the above control constraint (6) is not considered and $u_{d}=0$, an explicit solution can be obtained from minimization of the above quadratic problem (7) and (8) as follows:

$$
\begin{aligned}
& \min _{u} J_{w}=\left\|W_{u} u\right\|_{2}, \\
& \text { s.t. } \quad B_{f}^{*} u(t)=v(t),
\end{aligned}
$$

while the solution is given based on a weighted pseudoinverse as

$$
u(t)=W_{u}^{-1}\left(B_{f}^{*} W_{u}^{-1}\right)^{+} v(t) .
$$

where ' + ' is the pseudo-inverse operator. Obviously, there is no guarantee that the solution will satisfy the constraints.

Improved approaches have been proposed to accommodate to the limits. The Redistributed Pseudo-Inverse (RPI) method proposed by Virnig and Bodden (1994) is an alternative solution, in which all control inputs that violate their bounds in the pseudo-inverse solution are saturated and removed from the optimization. Then the control problem is solved with only the remaining control inputs as free variables. The Cascaded Generalized Inverse (CGI) method proposed by (Bordignon, 1996) is an iterative redistributed pseudo-inverse. All control inputs that violate their bounds are considered to be saturated values and are removed at each step. The redistribution process is continued until either the pseudo-inverse solution is feasible or all control inputs are saturated. Enns (1998) also suggests to compute the pseudo-inverse solution iteratively, as in the CGI, but only to saturate one control input per iteration. For the proposed approach and in order to assure the reliability of the actuators, the most reliable actuator will be saturated first at each step and then removed from the CGI optimization problem.

3.3. Reference governor post-fault formulation. The state description of the plant in a closed loop scheme, after the fault diagnosis, is given by

$$
\left\{\begin{aligned}
x(t+1) & =\Phi_{f} x(t)+G_{f} g(t), \\
y(t) & =H_{y} x(t), \\
c(t) & =H_{c}^{f} x(t)+L_{f} g(t),
\end{aligned}\right.
$$

where $\Phi_{f}$ and $G_{f}$ represent the global system dynamics after the fault occurrence:

$$
\begin{aligned}
& \Phi_{f}=\Phi+B \Gamma K, \\
& G_{f}=G-B \Gamma K_{g} .
\end{aligned}
$$

Consider the RG unit in the faulty case, with the following assumptions:

B1. $\Phi_{f}$ is a stable matrix.

B2. The system (34) is offset-free with respect to $g(t)$, i.e., $H_{y}\left(I_{n}-\Phi_{f}\right)^{-1} G_{g}^{f}=I_{p}$.

The solution minimizing the cost function (26) is

$$
g(t):=\arg \min _{g \in \mathcal{V}_{f}(x(t))} J(x(t), g(t), r),
$$

with $\mathcal{V}_{f}(x(t))$ being the set of the disturbance-free virtual evolution of the constraint vector $\bar{c}_{f}(k, x(t), g)$ after the fault occurrence,

$$
\begin{aligned}
& \mathcal{V}_{f}(x(t)) \\
& \quad=\left\{g \in \mathcal{W}_{\delta}^{f}: \bar{c}_{f}(k, x(t), g) \in \mathcal{C}_{k}^{f}, \forall k \in \mathbb{Z}_{+}\right\},
\end{aligned}
$$

where $\bar{c}_{f}(k, x(t), g)$ is given by

$$
\begin{aligned}
& \bar{c}_{f}(k, x(t), g) \\
& \quad=H_{c}^{f}\left(\Phi_{f}^{k} x(t)+\sum_{i=0}^{k-1} \Phi_{f}^{k-i-1} G_{f} g\right)+L_{f} g
\end{aligned}
$$

and

$$
\begin{aligned}
\mathcal{W}_{\delta}^{f} & :=\left\{g \in \mathbb{R}^{p}: \bar{c}_{g} \in \mathcal{C}_{f}^{\delta}\right\}, \\
\mathcal{C}_{f}^{\delta} & :=\mathcal{C}_{\infty}^{f} \sim \mathcal{B}_{\delta} .
\end{aligned}
$$

Here $\mathcal{C}_{\infty}^{f}$ is the prescribed constrained set after the fault occurrence, and $\mathcal{B}_{\delta}$ is a ball with radius $\delta$ centered at the origin. The following properties hold true for the above described RG in the faulty case.

Theorem 1. Let Assumption B1 be satisfied. Consider the system (34) along with the RG selection rule (37), and let $\mathcal{V}_{f}(x(0))$ be non-empty. Then we have the following:

1. The minimizer in (37) uniquely exists at each $t \in \mathbb{Z}_{+}$ and can be obtained by solving a convex constrained optimization problem, viz. the fact that $\mathcal{V}_{f}(x(0))=$ $\mathcal{V}\left(x\left(t_{f}\right)\right)$ is non-empty implies that $\mathcal{V}_{f}(x(t))$ is nonempty along the trajectories generated by the $R G$ command (34). Such a time of fault occurrence $t_{f}$ is determined by the diagnosis stage.

2. The set $\mathcal{V}_{f}(x(t))$ is determined for any, $x(t) \in$ $\mathbb{R}^{n}$ where there exists an integer $k_{0}^{f}$ such that, if $\bar{c}_{f}(k, x(t), g) \in \mathcal{C}_{k}^{f}, k \in\left\{0,1, \ldots, k_{0}^{f}\right\}$, then $\bar{c}_{f}(k, x(t), g) \in \mathcal{C}_{k}^{f}, \forall k \in \mathbb{Z}_{+}$. Such a constraint horizon $k_{0}^{f}$ can be determined off-line as described by Gilbert and Tan (1991).

3. The constraints are fulfilled for all $t \in \mathbb{Z}_{+}$. 
4. The overall system is asymptotically stable. In particular, whenever $r(t) \equiv r$, and $g(t)$ converges either to $r$ or to its best steady-state admissible approximation $\hat{r}$, with

$$
\hat{g}(t):=\hat{r}:=\arg \min _{g \in \mathcal{V}_{f}(x(t))} J(x(t), g(t), r) .
$$

Consequently, by the offset-free condition B2, we get $\lim _{t \rightarrow \infty} \bar{y}(t)=\hat{r}$, where $\bar{y}$ is the disturbance-free component of $y$.

\section{Flight control example}

The ADMIRE model has been used by several researchers (e.g., Harkegard, 2003) and within the Group of Aeronautical Research and Technology in Europe (GARTEUR). The linear model used here has been obtained at a low speed flight condition of Mach 0.22 at an altitude of $3000 \mathrm{~m}$ and is similar to the one by Harkegard and Glad (2005). The states are $x=[\alpha \beta p q r]^{T}$ with controlled outputs $y=[\alpha \beta p]$, where $\alpha$ is the angle of attack (rad), $\beta$ is the sideslip angle ( $\mathrm{rad})$, and $p$ is the roll rate $(\mathrm{rad} / \mathrm{s}), q$ defines the pitch rate $(\mathrm{rad} / \mathrm{s})$ and $r$ is the yaw rate $(\mathrm{rad} / \mathrm{s})$.

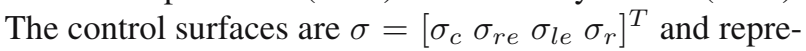
sent the deflections of the canard, right eleven, left eleven and rudder, respectively. Besides, the actuator position constraints are

$$
\begin{aligned}
\sigma_{\min } & =\left[\begin{array}{llll}
-55^{\circ} & -30^{\circ} & -30^{\circ} & -30^{\circ}
\end{array}\right]^{T}, \\
\sigma_{\max } & =\left[\begin{array}{llll}
25^{\circ} & 30^{\circ} & 30^{\circ} & 30^{\circ}
\end{array}\right]^{T} .
\end{aligned}
$$

A linearized model (Harkegard and Glad, 2005), for a sampling time of $0.5 \mathrm{~s}$, is

$$
\begin{aligned}
A & =\left[\begin{array}{crrrr}
1.0214 & 0.0054 & 0.0003 & 0.4176 & -0.0013 \\
0 & 0.6307 & 0.0821 & 0 & -0.3792 \\
0 & -3.4485 & 0.3979 & 0 & 1.1569 \\
1.1199 & 0.0024 & 0.0001 & 1.0374 & -0.0003 \\
0 & 0.3802 & -0.0156 & 0 & 0.8062
\end{array}\right], \\
B & =\left[\begin{array}{crrr}
0.1823 & -0.1798 & -0.1795 & 0.0008 \\
0 & -0.0639 & 0.0639 & 0.1397 \\
0 & -1.5840 & 1.5840 & 0.2936 \\
0.8075 & -0.6456 & -0.6456 & 0.0013 \\
0 & -0.1005 & 0.1005 & -0.4114
\end{array}\right]
\end{aligned}
$$

In this example, the actuator dynamics are neglected, and the approximate model can be given where

$$
B=B_{v} B^{*}
$$

and

$$
B_{v}=\left[\begin{array}{c}
0_{2 \times 3} \\
I_{3 \times 3}
\end{array}\right]
$$

Table 1. Failure rates of elementary components.

\begin{tabular}{|c|c|}
\hline \multicolumn{2}{|c|}{ Failure rates } \\
\hline \hline$\lambda_{1}^{0}$ & $4 \mathrm{e}-3 \mathrm{~min}^{-1}$ \\
\hline$\lambda_{2}^{0}$ & $9 \mathrm{e}-4 \mathrm{~min}^{-1}$ \\
\hline$\lambda_{3}^{0}$ & $3 \mathrm{e}-3 \mathrm{~min}^{-1}$ \\
\hline$\lambda_{4}^{0}$ & $9 \mathrm{e}-4 \mathrm{~min}^{-1}$ \\
\hline
\end{tabular}

$$
B^{*}=\left[\begin{array}{crrr}
0 & -1.5840 & 1.5840 & 0.2936 \\
0.8075 & -0.6456 & -0.6456 & 0.0013 \\
0 & -0.1005 & 0.1005 & -0.4114
\end{array}\right] \text {. }
$$

The resulting virtual control input $v(t)$ contains the angular accelerations in roll, pitch, and yaw produced by the control surfaces.

In order to illustrate the proposed approach in the short time window, we adapt the values of the actuator failure rates with the time of the discussed scenario. The failure rates are considered with a very huge value and given in Table 1.

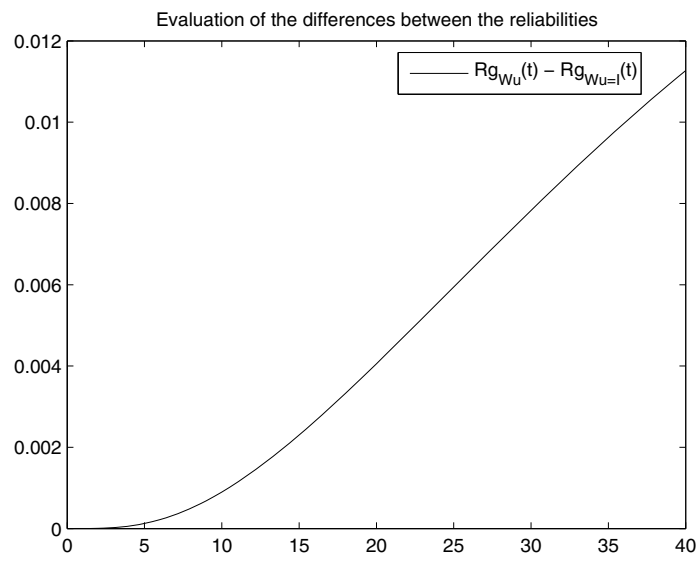

Fig. 4. Aircraft trajectory with re-allocation and the reference governor.

In this example, an optimal solution of the reconfigurable control problem is calculated in order to manage smartly the set of the actuators and increase the overall system reliability. The desired efforts are distributed against an optimal choice of the weighting matrix $W_{u}$ based on the reliability indicators (14). With the proposed choice, the most reliable actuators defined by small failure rate values are more solicited in the effort distribution.

Figure 5] shows simulation results when a partial loss of effectiveness control is considered. In the considered scenario, the partial losses of effectiveness control correspond to $\gamma_{3}=0.3$. As shown for this scenario, the control re-allocation strategy is sufficient to compensate the effect of the considered fault where the signal references are not modified and the system outputs track the references. Indeed, it can be seen clearly in Fig. 6 that the desired efforts 

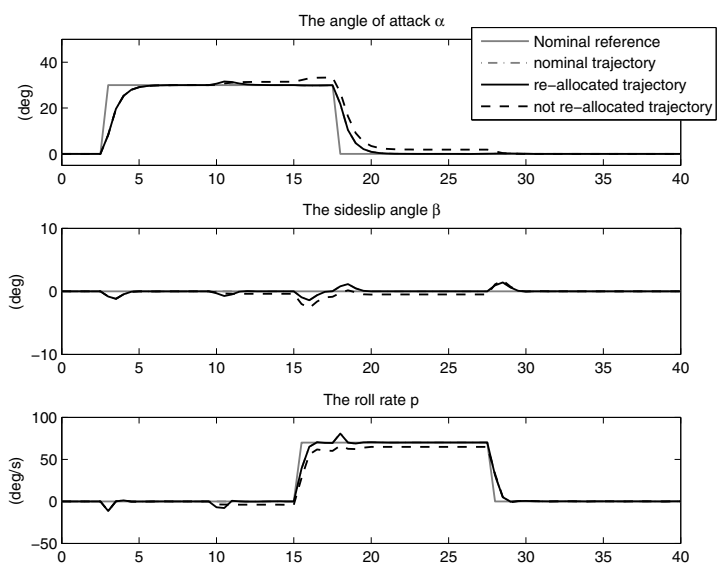

Fig. 5. Aircraft trajectory with re-allocation.

are distributed differently among the actuators taken into account the fault considered and the actuator reliability. This result can be justified by the fact that the analysed fault is relatively minor regarding the actuator availability.
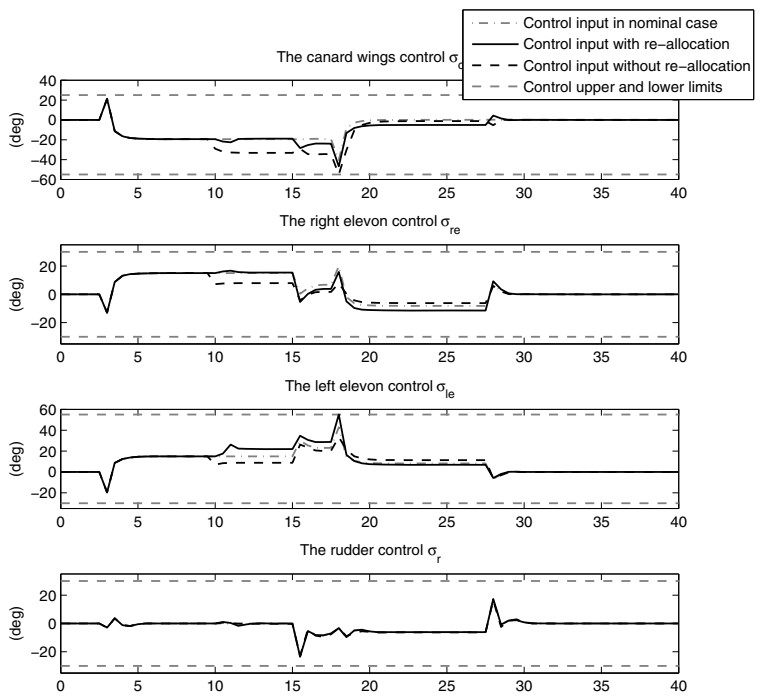

Fig. 6. Control input with re-allocation.

However, for a more sever degraded situation, the reference governor is needed to manage actuator saturation and tolerate the considered faults. Figure 7 shows the aircraft trajectories and the reconfigured references for $\gamma_{3}=0.85$. In fact, for this scenario the reference signals are modified in order to guarantee the system stability and distribute the desired efforts among the actuators with respect to system constraints. In Fig. 8, the efforts are distributed differently into account actuator reliability with respect to the examined fault and saturations.
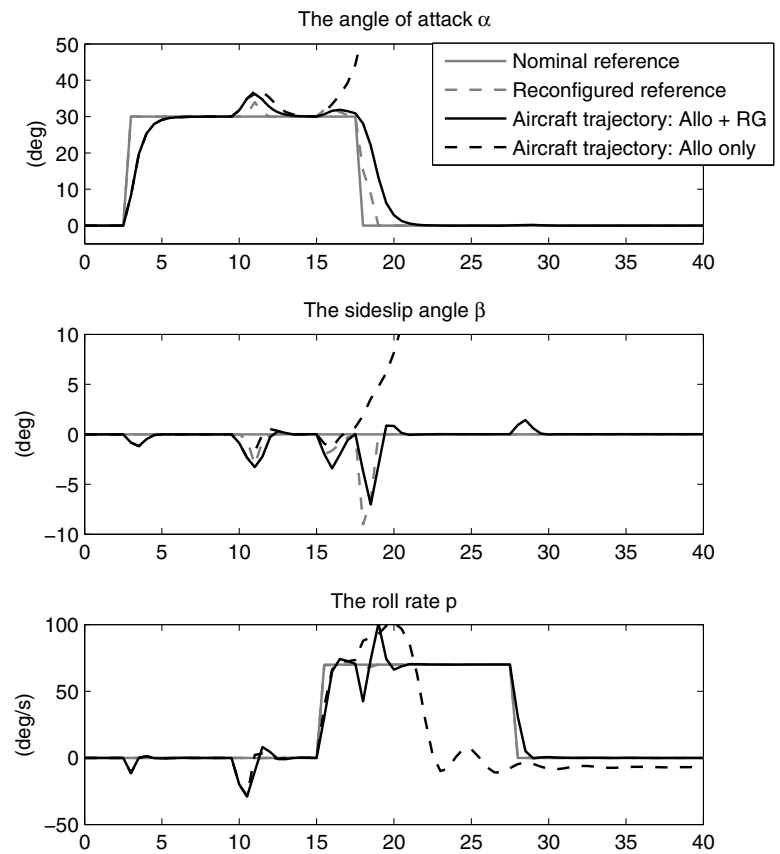

Fig. 7. Aircraft trajectory with re-allocation and the reference governor.
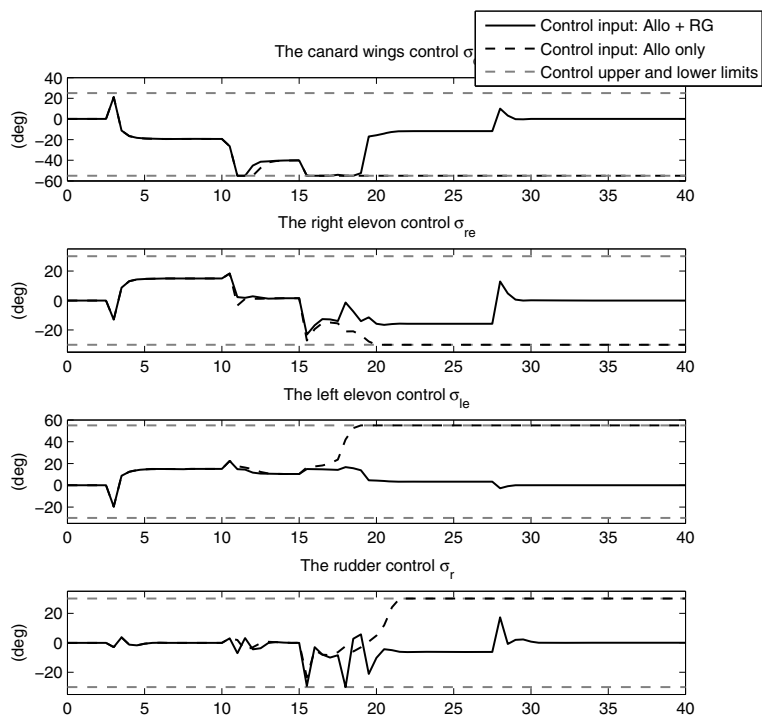

Fig. 8. Control input with re-allocation and the reference governor.

\section{Conclusion}

A contribution for an optimal reconfigurable control allocation strategy against reliability was proposed. The distribution of the desired efforts computed by the control law was considered based on actuator reliability. This paper presented a new approach to fault adaptation based on 
reference governor combined with reconfigurable control allocation. For minor faults, control re-allocation can be a reliable solution to distribute the desired efforts with respect to actuator reliability by an optimal choice of the weighing matrix. However, for severely degraded situations, the RG can be used to solve the problem of stability and saturation also with respect to actuator reliability. The RG unit could modify the references and add an offset to the control inputs after fault occurrence and constraint violation in order to adapt the dynamics to the new special behavior which corresponds to faulty mode functioning. The simulation results show that these methods might improve the system performance and ensure safe plant functioning. Moreover, an optimal choice of the weighing matrix was proposed based on the characteristic of the implemented actuators. This strategy can preserve and improve the availability of actuators during a mission with high overall system reliability.

\section{References}

Alwi, H. and Edwards, C. (2008). Fault tolerant control using sliding modes with on-line control allocation, Automatica 44(7): 1859-1866.

Angeli, D., Casavola, A. and Mosca, E. (2001). On feasible set-membership state estimators in constrained command governor control, Automatica 37(1): 151-156.

Bemporad, A., Casavola, A. and Mosca, E. (1997). Nonlinear control of constrained linear systems via predictive reference management, IEEE Transactions on Automatic Control 42(3): 340-349.

Blanke, M., Kinnaert, M., Lunze, J. and Staroswiecki, M. (2006). Diagnosis and Fault Tolerant Control, Control Systems Series, Springer-Verlag, London.

Bordignon, K. (1996). Constrained Control Allocation for Systems with Redundant Control Effector, Ph.D. thesis, Virginia Polytechnic Institute \& State University, Blacksburg, VA.

Boussaid, B., Aubrun, C. and Abdelkrim, M. (2010). Fault adaptation based on reference governor, Proceedings of the IEEE Conference on Control and Fault Tolerant Systems, SyStol'10, Nice, France, pp. 257-262.

Burken, J., Lu, P., Wu, Z. and Bahm, C. (2001). Two reconfigurable flight control design methods: Robust servomechanism and control allocation, Journal of Guidance, Control, and Dynamics 24(3): 482-493.

Casavola, A., Papini, M. and Franz, G. (2006). Supervision of networked dynamical systems under coordination constraints, IEEE Transactions on Automatic Control 51(3): 421-437.

Casavola, A., Franze, G. and Sorbara, M. (2007). Referenceoffset governor approach for the supervision of constrained networked dynamical systems, Proceedings of the European Control Conference, Kos, Greece, pp. 7-14.

Casavola, A. and Garone, E. (2010). Fault tolerant adaptive control allocation schemes for overactuated systems,
International Journal of Robust and Nonlinear Control 20(17): 1958-1980.

Cox, D. (1972). Regression models and life tables, Journal of the Royal Statistical Society, Series B 34: 187-220.

Durham, W. (1993). Constrained control allocation, Journal of Guidance, Control, and Dynamics 16(4): 717-125.

Enns, D. (1998). Control allocation approaches, AIAA Guidance, Navigation, and Control Conference, Boston, VA, USA, pp. 98-108.

Gertsbakh, I. (2000). Reliability Theory with Applications to Preventive Maintenance, Springer-Verlag, New York, NY.

Gilbert, E., Kalmanovsky, I. and Tan, K. (1995). Discrete-time reference governors and the nonlinear control of systems with state and control constraints, International Journal on Robust and Nonlinear Control 5(5): 487-504.

Gilbert, E. and Tan, K. (1991). Linear systems with state and control constraints: The theory and application of maximal output admissible sets, IEEE Transactions on Automatic Control 36(9): 1008-1020.

Guenab, F., Theilliol, D., Weber, P., Zhang, Y. and Sauter, D. (2006). Fault tolerant control design: A reconfiguration strategy based on reliability analysis under dynamic behavior constraints, Proceedings of 6th IFAC Safeprocess Symposium, Beijing, China, pp. 1033-1038.

Harkegard, O. (2003). Backstepping and Control Allocation with Applications to Flight Control, Ph.D. thesis, Linköping University, Linköping.

Harkegard, O. and Glad, S. (2005). Resolving actuator redundancyoptimal control vs. control allocation, Automatica 41(1): 137-144.

Johansen, T. and Johansen, T.A. (2008). Adaptive control allocation, Automatica 44(11): 2754-2765.

Kawakernaak, H. and Sivan, R. (1972). Linear Optimal Control Systems, Wiley-Interscience, New York, NY.

Khelassi, A., Theilliol, D. and Weber, P. (2009). Reconfigurability for reliable fault-tolerant control design, 7 th Workshop on Advanced Control and Diagnosis, Zielona Gora, Poland.

Kolmanovsky, I. and Sun, J. (2006). Parameter governors for discrete-time nonlinear systems with pointwise-in-time state and control constraints, Automatica 42(5): 841-848.

Martorell, S., Sanchez, A. and Serradell, V. (2009). Agedependent reliability model considering effects of maintenance and working conditions, Reliability Engineering and System Safety 64(1): 19-31.

Noura, H., Theilliol, D., Ponsart, J. and Chamssedine, A. (2009). Fault Tolerant Control Systems: Design and Practical Application, Springer, Dordrecht/Heidelberg/London.

Staroswiecki, M. (2003). Actuator faults and the linear quadratic control problem, Proceedings of the 42th IEEE Conference on Decision and Control, Maui, HI, USA, pp. 959-965.

Theilliol, D., Chemsseddine, A., Zhang, Y. and Weber, P. (2009). Optimal reconfigurable control allocation design based on reliability analysis, 7th IFAC Symposium on Fault Detection, Supervision and Safety of Technical Processes, Barcelona, Spain, (on CD-ROM). 
Theilliol, D., Join, C. and Zhang, Y. (2008a). Actuator fault tolerant control design based on a reconfigurable reference input, International Journal of Applied Mathematics Computer Science 18(4): 553-560, DOI: 10.2478/v10006-0080048-1.

Theilliol, D., Zhang, Y., Ponsart, J. and Aubrun, C. (2008b). Actuator fault tolerant control system with re-configuring reference input design based on MPC, Proceedings of the 6th Workshop on Advanced Control and Diagnosis, Coventry, UK, pp. 275-280.

Virnig, J. and Bodden, D. (1994). Multivariable control allocation and control law conditioning when control effectors limits, AIAA Guidance, Navigation, and Control Conference, Scottsdale, AZ, USA, pp. 572-582.

Zhang, Y., Suresh, V., Jiang, B. and Theilliol, D. (2007). Reconfigurable control allocation against aircraft control effector failures, IEEE International Conference on Control Applications, CCA 2007, Singapore, pp. 1197-1202.

Zhou, K., Doyle, J. and Glover, K. (1996). Robust and Optimal Control, Prentice Hall, Upper Saddle River, NJ.

Philippe Weber received his M.Sc. in automatic control and signal processing in 1995 from Henri Poincaré University in Nancy, France, and the Ph.D. degree in 1999 from the National Polytechnic Institute of Grenoble, France. He has been an assistant professor at the University of Lorraine since 2000, and a member of the Research Centre for Automatic Control of Nancy (CRAN), associated with the National Research Center of Science CNRS (UMR 7039). He focuses his interest on modeling problems in maintenance, prognosis and dynamic reliability. He develops fault-tolerant control systems including reliability analysis. Since 2000 his research interest has focused on modeling methods based on Bayesian networks.

Boumedyen Boussaid was born in 1972 in Tunisia. He received a Ph.D. in control engineering in 2011 from Henri Poincaré University and from the National School of Engineers of Tunis, and an engineering degree in 1997 in electrical engineering from the National School of Engineers of Tunis. Since 1999 he has been an assistant professor in the Department of Electrical Engineering at the High Institute of Technology, University of Gabs. He is currently a member of the Research Centre for Automatic Control of Nancy (CRAN) and the Research Unit on Modeling, Analysis and Control of Systems (MACS). His research interests focus on constrained control and fault tolerant control areas with application to wind turbines.
Ahmed Khelassi received his M.Sc. in automatic control and mechatronics for automotive and aerospace engineering in 2008 from the University of Bordeaux 1 (France), and a Ph.D. in automatic control from Henri Poincaré University (France) in 2011. His research works have been carried out at the Research Centre for Automatic Control of Nancy (CRAN), associated with the National Research Centre of Science (CNRS). His research interests include control systems, faulttolerant control, diagnosis, dependability and dynamic systems. In 2011 he joined the ArcelorMittal Maizieres Research Centre, where he works in the measurement and control group.

Didier Theilliol received the Ph.D. degree in control engineering from the University of Lorraine (France) in 1993. Since September 2004, he has been a full professor at the Research Center for Automatic Control of Nancy at the University of Lorraine, where he co-ordinates and leads national, European and international R\&D projects in steel industries, wastewater treatment plants, or the aerospace domain. His current research interests include model-based fault diagnosis method synthesis and active fault-tolerant control system design for LTI, LPV and multi-linear systems, and reliability analysis. He has published over 120 journal/conference papers and is a co-author of the book Fault-tolerant Control Systems: Design and Practical Applications (Springer, 2009). Didier Theilliol is a member of the editorial board of ISA Transactions and of the steering committee of the European Advanced Control and Diagnosis Working Group.

Christophe Aubrun received a Ph.D. in control engineering from Henri Poincaré University, France, in 1992. He is currently a member of the Research Centre for Automatic Control of Nancy (CRAN). Since 2005 he has been a professor in the Department of Electrical Engineering at the Institute of Technology, University of Lorraine. He has been involved in many projects with industry as well as European ones. His research interests lie in complex systems diagnosis and fault tolerant control areas with particular applications to water treatment processes and networked control systems. 